\title{
Non-surgical endodontic retreatment of extra-oral cutaneous sinus using propolis as an intracanal medicament: A case report
}

\author{
Fahad Quadri $^{1 *}$, Manjusha Warhadpande ${ }^{2}$ \\ ${ }^{1}$ Lecturer, ${ }^{2}$ Professor, ${ }^{1}$ CSMSS Dental College, Aurangabad, Maharashtra, ${ }^{2}$ Government Dental College, Nagpur, Maharashtra, India
}

*Corresponding Author: Fahad Quadri

Email: dr.fquadri@gmail.com

\begin{abstract}
This case report describes a successful non-surgical endodontic retreatment of the lower right central incisor with periapical lesion with tender extra-oral cutaneous sinus at the mental region. The extra-oral cutaneous sinus was misdiagnosed as acne \& was treated by a skin specialist. The tooth underwent root canal treatment in which Indian Propolis was used as an intracanal medicament. The tooth was obturated after 7 months after initiation of therapy. At 12 months recall intraoral periapical radiograph showed healing of the periapical lesion \& the extra-oral cutaneous sinus was healed with scarring. A thorough clinical evaluation, proper diagnosis and multidisciplinary treatment protocol are important for the success of the treatment. This paper suggest that Indian Propolis was effective in the healing of periapical lesion with extra oral sinus.
\end{abstract}

Keywords: Extra-oral sinus, Indian Propolis, Intracanal medicament \& Periapical lesion.

\section{Introduction}

An extra-oral sinus is normally the consequence of longstanding chronic infection due to caries, trauma, or more rarely periodontal infection or disease..$^{1-3}$ Misdiagnosing of extra-oral cutaneous sinus tracts of odontogenic origin is quite common due to the unexpected appearance of the lesion without historic odontogenic pain, discomfort, or cellulitis. ${ }^{3}$ A thorough examination of the lesion along with the radiographs are necessary for a specific diagnosis. ${ }^{4}$ Intracanal medicaments forms a part of endodontic disinfection during root canal treatment. Propolis which is extract from honey bees has a wide variety of use in dentistry including anti-caries agent, interim transport media for avulsed teeth, intra-canal medicament, Intra-canal irrigant, pulp capping agent, adjunct to radiotherapy, reduction of dentinal hypersensitivity, etc. ${ }^{5}$ In recent studies it has been shown that propolis is more effective against resistant microbes like E. faecalis \& is biocompatible to the peri-radicular tissues when compared with existing intracanal medicaments. ${ }^{6-7}$ This paper presents a retreatment case of extra-oral cutaneous sinus of odontogenic origin which was treated using Propolis as an intracanal medicament.

\section{Case Report}

A 25 years old female was referred to the Department of Conservative Dentistry \&Endodontics, Dental College \& Hospital, for pain with \#41 and swelling with pain in the chin region since 7 days (Fig. 1a). Dental history revealed that root canal therapy with respect to \#41 was performed 5 years back, but with time the symptoms (dull pain \& pus discharge) were not relieved and re-root canal treatment was performed which was incomplete due to missed appointment by the patient. Patient revealed initially around 3 years back single vesicle started to appear with serous discharge. The patient underwent treatment for vesicle which she thought was an acne under a dermatologist who prescribed her Tretinoin for 3 months. The vesicle used to subside after medication and used to reoccur after cessation of Tretinoin. Within some days the vesicle matured to form a puckering. Clinical extra-oral examination revealed a single non-discharging sinus at mentum with $1.5 \times 1 \mathrm{~cm}$ in dimensions, with surrounding induration which was tender, oedema and puckering to underlying skin (Fig. 1a). Clinical examination revealed dislodged coronal restoration with \#41 which also showed no response to electric pulp vitality test (C PULSE pulp tester, FOSHAN COXO Medical Instruments Co. Ltd.). IOPA radiograph revealed radiolucent lesion of 1 x $1 \mathrm{~cm}$ suggestive of chronic periapical infection (Fig. 1b). After a careful consideration and patients consent for the use of Propolis as medicament $\&$ it was decided to do retreatment \& observation. After isolating the tooth using rubber dam (Hygienic, Coltene/ Whaledent In., Mahwah. NJ) only single root canal as found on scouting, the working length was determined using Ingle \& Backland (2002) technique (Fig. 1c). Cleaning \& shaping of canal was done by step back technique \& apical enlargement of canal was done up-to ISO (International standard organization) size no. 50 using K-files (Mani, Japan) in a single appointment. Sodium hypochlorite $2.5 \%$ followed by Normal saline were used as irrigating solutions and were used along with syringe \& side vented needle (30 gauge, CERKAMED, Poland) during the cleaning \& shaping procedure. Indian propolis $(30 \%$ ethanol extract, Hi-tech India naturals LTD, New Delhi) was placed with a syringe with the help of side vented needle (30 gauge, CERKAMED, Poland) till the canal was completely filled. The tip of the needle was kept $1 \mathrm{~mm}$ short of the apex. Cotton pellet was placed over Temporary dressing Cavit (ESPE America, INC., Norristown, PA) with a minimum thickness of $4 \mathrm{~mm}$ for adequate seal of the access cavity and patient was recalled after 3 days to renew the medicament. The previous medicament was removed using saline irrigation and new dressing was placed. The intra-oral and extra-oral symptoms were relieved after the $1^{\text {st }}$ appointment. The medicament was changed three times and later it was 
planned for obturation. The patient missed her appointment for obturation and came back after 7 months for the continuation of the treatment, at this stage the tooth was asymptomatic with intact temporary restoration (Cavit) with periapical radiograph suggested a partial resolution of the lesion when compared to the original size of lesion. The tooth was obturated on the same day by lateral compaction technique \& the access cavity was sealed using Glass Ionomer cement (GC fuji II, Japan) (Fig. 1c). The tooth was asymptomatic with good periapical healing on radiograph $\&$ extra-oral depressed atrophic scarring was evident after 1year recall evaluation of lesion.

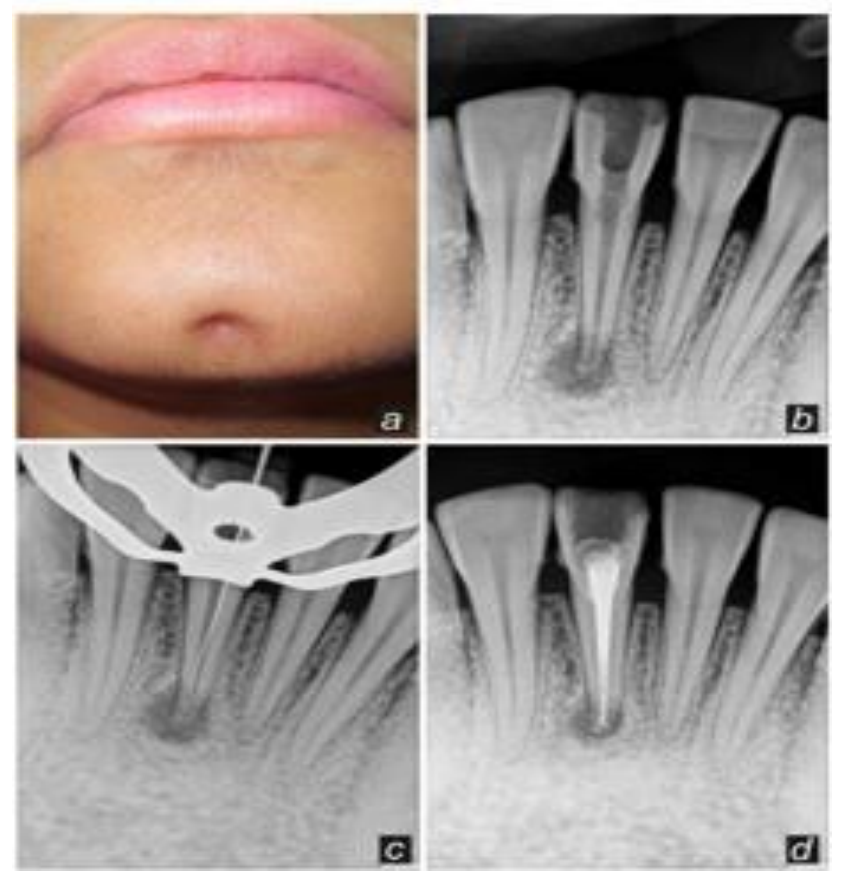

Fig. 1: (a) Extra oral image showing cutaneous sinus with surrounding diffuse swelling; (b): Preoperative radiograph of tooth \#41 showing chronic periapical lesion; (c): Working length radiograph of \#41; (d): Post-obturation radiograph o \#41 showing resolution of periapical lesion.

\section{Discussion}

A sinus tract is an abnormal channel that originates or ends in one opening. An orofacial fistula is a pathologic communication between the cutaneous surface of the face and the oral cavity. The terms sinus and fistulas are often used interchangeably. ${ }^{8}$

The purulent exudate of the odontogenic infection will have an extra-oral opening if the apices of the maxillary teeth are above the maxillary muscle attachments, and for the mandibular teeth below the mandibular muscle attachments. 1

Healing of long standing periapical lesion is the intimidating aspect of the endodontic therapy. In view of the potential for misdiagnosis of subcutaneous extraoral sinus, it is important that a full detailed history and comprehensive dental examination is completed to eliminate an odontogenic cause of the lesion. ${ }^{8-9}$ Clinically, a cutaneous sinus tract may mimic the appearance of an infected pimple, nodule, ulcer or an indurate cystic area on the skin \& the patient may seek advice initially from medical colleagues, assuming wrongly that the lesion is related to the skin. ${ }^{8-10} \mathrm{~A}$ misdiagnosed case often leads to unnecessary investigations and treatment protocols. ${ }^{11-12}$

Adequate measures which are used to eliminate the intracanal microorganisms would ensure a complete healing of the extra-oral sinus along with the periapical lesion. Intracanal medicaments have been thought as an essential step in killing the bacteria in root canals. ${ }^{6}$ The most fundamental therapy that is an important adjunct to root canal therapy is intracanal medicament and it is considered as an important mainstay that helps reduce the microbial flora beyond the levels previously achieved at the time of canal preparation. Intracanal medicaments are particularly useful in those areas which are not negotiated by instruments or irrigants as they penetrate these areas. These intracanal medicaments have the lasting effect between the appointments \& they ensure the reduction in the risk of proliferation and reorganisation of the residual bacteria that can cause reinfection of the root canals. The additional benefits of the intracanal medicaments are as follows: They provide relief from pain, exudate control in weeping canals, and boost apical healing. ${ }^{13}$ The gold standard in intracanal medicament in the practice of endodontics is calcium hydroxide which on a stand-alone basis performs most of the requisite functions and is considered as an ideal intracanal medicament. ${ }^{14}$ Calcium hydroxide has been shown to be less effective against Enterococcus faecalis and Candida albicans. ${ }^{15}$ Controversies on biocompatibility of calcium hydroxide especially when extruded into the periapical region is ambiguous with some reports endorsing its tolerance ${ }^{16-17}$ and some rejecting it. ${ }^{18-19}$ Hence, there is a definite need for a newer improved intracanal medicament that will aid in the success of root canal treatment.

Propolis is a mixture of resinous substances collected from plants by honey bees. The anti-microbial, antiinflammatory and immunomodulatory effect are well documented in the literature..$^{6-7}$ Hence Propolis was used in the present study as an intracanal medicament. The quality of Propolis depends on the geographic region from where Propolis is collected by the honey bees. ${ }^{5}$ Propolis has been shown to suppress the production of lipoxygenase and cyclooxygenase and inhibit in vivo the elevated production of leukotrienes B4 (LTB4) and leukotrienes C4 (LTC4). Caffeic acid present in propolis presents immunosuppressive activity, inhibiting the early and late events of $\mathrm{T}$ cell activation and the consequent release of cytokines such as IL-2 (Marquez et al., 2004) in an unspecific way of inhibition of ion channels (Nam et al., 2009). ${ }^{20}$ Propolis exhibits its antimicrobial action by breaking down the cytoplasm and preventing cell division (Oncag et. al 2008 \& Al Qathami \& Al- Madi 2003). ${ }^{21-22}$

Madhubala et al. (2011) showed that $100 \%$ elimination of E. faecalis after 2 days with propolis as an intracanal medicament, hence in the present case propolis was renewed after 3 days. $^{23}$ It is proven that ethanol component did not 
influence the antimicrobial action of propolis. ${ }^{24}$ The coronal seal also contributes to the success of endodontic treatment by preventing the micro-leakage in the root canal. An interim restoration of $4 \mathrm{~mm}$ thickness was chosen confirming to previous reports which also provided a good seal and prevented ingress of the bacteria from the oral cavity. ${ }^{25}$

In the present case, long term success has been reported \& ethanol extract of propolis has shown promising results in terms of the clinical and radiographic success of root canal treatment.

\section{Conclusion}

Indian origin Propolis (Indian Propolis) demonstrated good periapical healing in this present case report. Cutaneous facial sinus tracts of dental origin are often initially misdiagnosed and inappropriately treated \& hence its correct diagnosis and treatment by using effective intracanal medicament (Propolis) will result in predictable and rapid healing of these lesions.

\section{Source of funding}

None.

\section{Conflict of interest}

None.

\section{References}

1. McWalter G, Alexander J, del Rio C, Knott W. Cutaneous sinus tracts of dental aetiology. Oral Surg Oral Med Oral Pathol 1988;66:608-14.

2. Johnson BR, Remeikis NA, Van Cura JA. Diagnosis and treatment of cutaneous sinus tracts of dental origin. $J$ Am Dent Assoc 1999; 130:832-6.

3. Brown R, Jones R, Feimster T, Sam F. Cutaneous sinus tracts (or emerging sinus tracts) of odontogenic origin: a report of 3 cases. Clin Cosmet Investig Dent 2010;2:63-7.

4. Ørstavik D, Pitt Ford TR. Apical periodontitis: microbial infection and host responses. Essential endodontology. Prevention and treatment of apical periodontitis. Oxford: Blackwell Science. 1998:1-8.

5. Sardana D, InduShekar KR, Manchanda S, Saraf BG, Sheoran $\mathrm{N}$. Role of propolis in dentistry: review of the literature. Focus on Alternative and Complementary Therapies. 2013;18(3):11825.

6. Kandasamy D, Venkateshbabu N, Gogulnath D, Kindo AJ. Dentinal tubule disinfection with $2 \%$ chlorhexidine gel, propolis, morinda citrofolia juice, $2 \%$ povidone Iodine and calcium hydroxide. Int Endod J 2010;43:419-23.

7. Awadeh L, Bertarm AL, Hammad M. Effectiveness of propolis and calcium hydroxide as a short term intracanal medicament against Enterococcus faecalis: a laboratory study. Aust Endod J 2008;34:1-9.

8. Tidwell E, Jenkins JD, Ellis CD, Hutson B, Cederberg RA. Cutaneous odontogenic sinus tract to the chin: a case report. Int Endod J 1997;30:352-5.

9. Yang Z, Lai Y. Healing of a sinus tract of periodontal origin. A case report. J Endod 1992;18(4):178-80.

10. Barrowman R, Rahimi M, Evans M, Chandu A, Parashos P. Cutaneous sisnus tracts of dental origin. MJA 2007;186:264-5.

11. Sammut S, Malden N, Lopes V. Facial cutaneous sinuses of dental origin - a diagnostic challenge. BDJ 2013;215:555-8.
12. Pasternak-Júnior B, Teixeira CS, Silva-Sousa YT, Sousa-Neto MD. Diagnosis and treatment of odontogenic cutaneous sinus tracts of endodontic origin: three case studies. Int Endod $J$ 2009;42(3):271-6.

13. Siqueira JF JR, Lopes HP. Mechanisms of antimicrobial activity of calcium hydroxide: A critical review. Int Endod $J$ 1999;32:361-9.

14. Ghatole K, Gowdra RH, Azher S, Sabharwal S, Singh VT, Sundararajan BV. Enhancing the antibacterial activity of the gold standard intracanal medicament with incorporation of silver zeolite: An in vitro study. J Int Soc Prev Community Dent 2016;6(1):75.

15. Mohammadi Z, Dummer PMH. Properties and applications of calcium hydroxide in endodontics and dental traumatology. Int Endod J 2011;44:697-730.

16. Martine Guigand, Pascal Pellen-Mussi, Anne Le Goff, JeanMarie Vulcain, Martine Bonnaure-Mallet, "Evaluation of the cytocompatibility of three endodontic materials". J Endod 25(6):419-23.

17. Martin DM, Crabb HS. Br Dent J 1977;3,142(9):277-83.

18. Hauman $\mathrm{CH}$, Love RM. Biocompatibility of dental materials used in contemporary endodontic therapy: a review" Part 1. Intracanal drugs and substances. Int Endod J 2003;36:75-85.

19. Blomlöf L, Lindskog S, Hammarstrom L. Influence of pulpal treatments on cell and tissue reactions in the marginal periodontium. J Periodontol 1988;59:577-83.

20. Araujo MA, Libério SA, Guerra RN, Ribeiro MN, Nascimento FR. Mechanisms of action underlying the anti-inflammatory and immunomodulatory effects of propolis: a brief review. Rev Bras de Farmacognosia 2012;22(1):208-19.

21. Oncag O, Cogulu D, Uzel A, Sorkun K (2008). Efficacy of propolis as an intracanal medicament against Enterococcus faecalis. Gen Dent 54(5):319-22.

22. Qathami HA, Al-Madi E. Comparison of sodium hypochlorite, propolis and saline as root canal irrigants: A pilot study. Saudi Dent J 2003;15(2):100-3.

23. Madhubala MM, Srinivasan N, Ahamed S. Comparative evaluation of propolis and triantibiotic mixture as an intracanal medicament against Enterococcus faecalis. J Endod 2011;37(9):1287-9.

24. Flaviana BAF, Sergio AT, Odilla PSR, Cludio MF. Antimicrobial effect of propolis and other substances against selected endodontic pathogen. Oral Surg Oral Med Oral Pathol Radiol Endod 2007;104:709-16.

25. Aminozarbian MG, Feizianfard M, Karimi M. Sealing ability of three temporary filling materials in endodontically-treated teeth. Iranian Endodontic Journal 2009;4:1-4. Shahi S, Samiei $\mathrm{M}$, Rahimi S, Nezami H. In vitro comparison of dye penetration through four temporary restorative materials. Iran Endod J 2010;5:59-63.

How to cite this article: Quadri F, Warhadpande M.
Non-surgical endodontic retreatment of extra-oral
cutaneous sinus using propolis as an intracanal
medicament: A case report. Int J Oral Health Dent
2019;5(3):140-2.

